\title{
Immune profile in relation to sex steroid cyclicity in healthy women and women with multiple sclerosis
}

Per Kempe, Daniel Eklund, Agnes Hallin, Mats Hammar, Tomas Olsson, J an Brynhildsen and J an Ernerudh

The self-archived postprint version of this journal article is available at Linköping University Institutional Repository (DiVA):

http:// urn.kb.se/ resolve?urn=urn:nbn:se:liu:diva-147812

N.B.: When citing this work, cite the original publication.

Kempe, P., Eklund, D., Hallin, A., Hammar, M., Olsson, T., Brynhildsen, J ., Ernerudh, J ., (2018),

Immune profile in relation to sex steroid cyclicity in healthy women and women with multiple

sclerosis, J ournal of Reproductive Immunology, 126, 53-59. https:/ / doi.org/ 10.1016/j.jri.2018.02.006

Original publication available at:

https:// doi.org/ 10.1016/j.jri.2018.02.006

Copyright: Elsevier

http:// www.elsevier.com/

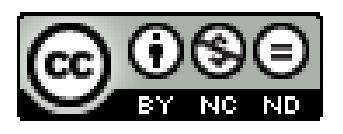




\section{Immune profile in relation to sex steroid cyclicity in healthy}

\section{women and women with multiple sclerosis}

Per Kempe (MD, PhD) $)^{1,2}$ Daniel Eklund $(\mathrm{PhD})^{3}$, Agnes Hallin ${ }^{3}$, Mats Hammar $(\mathrm{MD}, \mathrm{PhD})^{1}$, Tomas Olsson (MD, PhD) $)^{4}$, Jan Brynhildsen (MD, PhD) ${ }^{1^{*}}$ and Jan Ernerudh (MD, PhD) $)^{5^{*}}$

1. Obstetrics and Gynaecology, Department of Clinical and Experimental Medicine, Linköping University, SE58185, Linköping, Sweden.

2. Department of Obstetrics \& Gynaecology, County Hospital Sundsvall, SE-85643, Sundsvall, Sweden.

3. Department of Clinical and Experimental Medicine, Linköping University, SE-58185, Linköping, Sweden.

4. Department of Clinical Neuroscience, Karolinska Institutet, SE-17177, Stockholm, Sweden.

5. Clinical Immunology and Transfusion Medicine, Department of Clinical and Experimental Medicine, Linköping University, SE-58185, Linköping, Sweden

* Equal contribution

\section{Corresponding author:}

Jan Brynhildsen

Obstetrics and Gynaecology, Department of Clinical and Experimental Medicine, Linköping University, SE-58185, Linköping, Sweden

Tel: +46101033132

e-mail: jan.brynhildsen@liu.se 


\begin{abstract}
To prospectively study systemic in vivo immunological effects of sex hormones, using different phases of oral combined hormonal contraceptives (CHC), and the natural menstrual cycles in both healthy women and in women with multiple sclerosis (MS), blood samples from sixty female MS patients and healthy controls with and without CHC were drawn in high and low estrogenic/progestogenic phases. Expression of Th-associated genes in blood cells was determined by qPCR and a panel of cytokines and chemokines was measured in plasma. High hormone level phases were associated with increases in Th1 (TBX21) and Th2 (GATA3) associated markers, as well as the B cell-associated chemokine CXCL13, while the inhibitory regulator CTLA-4 was decreased. These changes were not observed in MS patients, of whom most were treated with immunomodulatory drugs. Our data indicate immune activating properties in vivo of high steroid sex hormone levels during both $\mathrm{CHC}$ and normal menstrual cyclicity.
\end{abstract}

\title{
Key words
}

Contraception, Immunology, Estrogen, Progesterone, Multiple sclerosis, Menstrual cycle 


\section{Introduction}

Several clinical observations indicate sex-differences in immune responses. In particular, women show stronger responses to vaccinations, lower susceptibility to some infections, and are at a higher risk for several autoimmune diseases (Klein and Flanagan 2016) including multiple sclerosis (MS) (D'Hooghe M et al. 2013, Harbo et al. 2013), altogether indicating a stronger immune reactivity in women compared with men. Since these phenomena are more evident in the post-pubertal and premenopause period (Klein and Flanagan 2016) it is likely that levels of sex steroids, at least in part, explain the stronger immune responses in women. On the other hand, variations in sex hormone levels are also involved in attenuation of immune responses, leading to increased severity of some infections during the third trimester of pregnancy (Kourtis et al. 2014) as well as improvement of inflammatory diseases like MS during the same period (Bernardi et al. 1991, Confavreux et al. 1998). Furthermore, some studies suggest a beneficial role of sex hormones in MS patients during parts of the normal menstrual cycle (Zorgdrager and De Keyser 1997, Zorgdrager and De Keyser 2002), while other studies find no differences in MS symptoms during the menstrual cycle (Pozzilli et al. 1999, Holmqvist et al. 2009).

The effects of sex hormones have also been studied in relation to external sex steroid exposure. In the animal model of MS, experimental autoimmune encephalomyelitis (EAE), estrogens were reported to have beneficial effects, attenuating inflammation and clinical symptoms when administrated as (1) 17ß-estradiol (E2) (Polanczyk et al. 2003), (2) selective estrogen receptor $\alpha(E R \alpha)$ ligand or the synthetic estrogen ethinylestradiol (EE) (Morales et al. 2006, Klein and Flanagan 2016). In humans, one phase I/II study in women with MS suggested that treatment with estriol (E3), which reaches high concentrations only during pregnancy, had beneficial effects on contrast-enhancing MRI lesions (Sicotte et al. 2002), while another recent placebo-controlled, double blind, phase II trial of estriol, as an add-on to glatiramer acetate, failed to convincingly show a beneficial effect; relapse rate decreased, but MRI activity did not (Voskuhl et al. 2016). In line with a beneficial effect of estrogens, a lower MS incidence (Alonso et al. 2005), and a more benign disease course (Sena et al. 2012, Gava 
et al. 2014), were reported among users compared with non-users of combined hormonal contraceptives (CHC). Also, we reported that $\mathrm{CHC}$ use was associated with a higher age at onset of MS (Holmqvist et al. 2010). However, in other studies of hormonal contraceptives (although type of hormonal contraceptive was not specified) the incidence of MS was not affected (Thorogood and Hannaford 1998, Hernan et al. 2000), and in primary progressive (PP) MS, use of hormonal contraceptives was rather associated with a more severe disease (D’hooghe et al. 2012).

Taken together, several lines of evidence show that sex hormones affect immune reactivity, although the direction seems to depend on the context. Sex hormones, like estrogens, act on immune cells by binding to intra- and extra cellular receptors. T helper (Th) cells and their polarisation into different Th subsets are of crucial importance for regulation of immunity, both in pregnancy (Saito et al. 2010, Ernerudh et al. 2011) and in MS/EAE (Merrill et al. 1992, Edström et al. 2011). Consequently, Th subset regulation has been addressed in studies of estrogen-mediated immunological effects. In EAE, studies indicate that estrogens decrease disease-promoting Th1 and increase the presumed beneficial Th2 and regulatory T cell (Treg) responses (Morales et al. 2006, Polanczyk et al. 2006). In humans, similar findings have been reported (Faas et al. 2000, Lee et al. 2010). In contrast, several reports showed that estrogens upregulated Th1 immunity (Maret et al. 2003, Karpuzoglu et al. 2007). In addition, augmentation of B cell responses by estrogens have been described (Paavonen et al. 1981). The diverging results can be explained by study objects and study design (experimental models versus human studies, in vitro versus in vivo, methodological issues). Furthermore, in humans there are several confounding factors that are at play and there is a big inter-individual variation in immune parameters. The aim of the present prospective study was to investigate if high and low sex steroid hormone states during the menstrual cycle and during use of combined hormonal contraceptives have in vivo effects on Th related transcription factors, cytokines and chemokines in healthy women and women with MS. To avoid inter-individual variation, we used a paired-sample design with blood samples from the same woman obtained at high and low sex steroid levels, respectively.

\section{Materials and methods}




\subsection{Subjects}

\subsubsection{Healthy women}

Fifteen healthy women using CHC and 15 healthy women without hormonal contraception and with regular menstrual cycles, all 18-45 years old, were included. These women were recruited among students and employees at Linköping University Hospital, Sweden (Table 1). The exclusion criteria were inflammatory diseases or other diseases affecting the central nervous system (CNS), ongoing or planned pregnancy, other hormonal contraception than CHC, anti-depressive medication, mental or language difficulties. Of the healthy controls using CHC, nine women were on monophasic regimens with $30 \mu \mathrm{g}$ EE daily, one woman was on a triphasic regimen with 30-40 $\mu$ g EE daily, two women were on a monophasic regimen with $20 \mu \mathrm{g}$ EE daily, and one woman used a vaginal ring. Four different progestogens were used in combination with EE.

\subsubsection{Women with $M S$}

Women with a definitive diagnosis of MS according to the revised McDonald criteria (Polman et al. 2011), 18 to 40 years old, were recruited at the Departments of Neurology at the University Hospital of Linköping and the Karolinska University Hospital of Stockholm, both in Sweden (Table 1).

Exclusion criteria were: Expanded Disability Status Scale (Kurtzke 1983) $($ EDSS) > 6.5, clinical exacerbation of MS during the last three months, other inflammatory diseases or other diseases affecting CNS, ongoing or planned pregnancy, other hormonal contraception than CHC, antidepressive medication, mental or language difficulties. Women using transdermal or vaginal administration of combinations of EE and progestogen were also allowed to participate in the study and were labelled as CHC users. Of the MS patients using $\mathrm{CHC}$, eight women were on monophasic regimens with $30 \mu \mathrm{g}$ EE daily, one woman was on a monophasic regimen with $20 \mu \mathrm{g}$ EE daily, one woman was on a triphasic regimen with $35 \mu$ g EE daily, one woman used a vaginal ring, and one woman used a transdermal patch. Six different progestogens were used in combination with EE. MS patients were allowed to use immunomodulatory medication during the study period as long as it was unchanged during the study period of three months. If a patient had an exacerbation of MS or had to 
use corticosteroids or antibiotics during the study period she was excluded. The most recent Multiple Sclerosis Severity Score (MSSS) (Roxburgh et al. 2005) was collected from the medical record of each woman included.

\subsection{Blood sampling and study design}

CHC used by the subjects consisted of 21 days of EE in combination with a progestogen and 7 days of placebo in a 28 day cycle. Cycle day 1 was set to the first pill-day for the CHC users and first day of bleeding for the women without hormonal contraception. Paired blood sampling was performed on cycle day 20 or 21 (longest possible high estrogen/progestogen exposure) and day 27 or 28 (longest possible low estrogen/progestogen exposure) for CHC-users. For women without hormonal contraception, paired blood sampling was performed on cycle day 5, 6, or 7 (longest possible low estrogen/progestogen exposure) and day 26, 27, or 28 (longest possible high estrogen/progestogen exposure). The two samples from each woman were drawn during the same cycle.

To capture ongoing in vivo activity, blood was drawn directly into PAXgene tubes (PreAnalytix, GmbH, Hombrechtikon, Switzerland), with immediate stabilisation of intracellular RNA. The PAXgene tubes were handled according to the manufacturer's instruction and frozen in $-70^{\circ} \mathrm{C}$ until analysis. In addition, an EDTA plasma tube was obtained and centrifuged within 1 hour, followed by aliquoting to $0.5 \mathrm{~mL}$ cryo tubes that were frozen in $-70^{\circ} \mathrm{C}$. From the women who were not using $\mathrm{CHC}$, blood samples at each occasion were assayed for concentrations of 17ß-estradiol (E2) and progesterone using the routine methods at the accredited Departments of Clinical Chemistry of the University Hospital, Linköping, Sweden and Karolinska University Hospital, Solna, Sweden.

\subsection{Extraction of $m R N A$, conversion to $c D N A$ and $q P C R$}

RNA was extracted from whole blood using the PAXgene system according to the manufacturer's instructions, before converted into cDNA using the High Capacity cDNA Synthesis Kit (Applied Biosystems, Foster City, CA, USA). The cDNA synthesis was carried out on an Arktik ${ }^{\mathrm{TM}}$ Thermal 
Cycler (Thermo Fisher Scientific, Waltham, MA, USA). mRNA expression was quantified using the Applied Biosystems 7500 Fast Real-Time PCR System (Applied Biosystems, Foster City, CA, USA). cDNA amplification was performed using TaqMan Universal PCR Master Mix, no AmpErase UNG (Applied Biosystems). For quantification of cDNA a five-point serially four-fold diluted standard curve was developed from peripheral blood mononuclear cell cultures stimulated with phytohaemagglutinin. The mRNA expression of the $\mathrm{T}$ cell-associated genes was standardized to the $\mathrm{T}$ cell-specific reference gene $C D 3 E$, and results expressed as ratios. $C D 3 E$ was used as a reference gene since the ratio of $C D 3 E$ to the generally used reference gene $18 S$ changed with high/low estrogen/progestogen phases, indicating fluctuations in T cells numbers. Expression of CD3E has been shown to be stable at physiological stimulations (anti-CD3/CD28 stimulation) and in in vivo situations of systemic inflammation (for example in preeclampsia), and the use of CD3E was shown to normalize the expression relative to the T cell content in the sample (Edström, Ernerudh et al., data to be published).

To assess expression of Th subset and regulatory gene expression in blood cells, TaqMan Gene Expression Assay (Applied Biosystems) was used for Th1-associated TBX21 (Hs00203436_m1), Th17-associated RORC (Hs01076112_m1) and immune regulatory CTLA-4 (Hs03044418_m1), while previously optimized in-house primers and probes (purchased from Eurogentec, Seraing, Belgium) were used for analysing reference gene 18S, Treg-associated FOXP3, Th2-associated GATA3 expression (Edström et al. 2011) and T cell-specific reference gene CD3E (Edström, personal communication) expression. All samples were analysed in duplicate and an acceptable level of variation was set to a coefficient of variance $<15 \%$. The intra-assay variance was $4.9 \%$ for $18 S, 4.9 \%$ for CD3E, $5.6 \%$ for FOXP3, $4.8 \%$ for GATA3, $4.7 \%$ for RORC, $5.7 \%$ for TBX21 and $4.7 \%$ for CTLA-4.

\subsection{Measurement of cytokines and chemokines in plasma}


To assess the effect of cyclic hormone levels on levels of cytokines and chemokines in plasma, we used multiplex bead technology (MILLIPLEX ${ }^{\circledR}$ MAP Kit, Human Cytokine/Chemokine Magnetic Bead Panels Cat. \#: HCYTOMAG-60K-11, HCYP2MAG-62K-02 and HCYP3MAG-63K-03) according to the manufacturer's description. Analysed cytokines and chemokines included (lowest detection limit in brackets): GM-CSF (1.6 pg/ml), IL-13 (1.6 pg/ml), IL-17A (0.8 pg/ml) IL-1RA (1.6 pg/ml), CXCL10 (8.0 pg/ml), IL-6 (0.8 pg/ml), IFN- $\gamma$ (0.8 pg/ml), IL-10 (1.6 pg/ml), CXCL8 (0.8 pg/ml), CXCL1 (8.0 pg/ml), CCL22 (8.0 pg/ml), CXCL13 (2.0 pg/ml), CCL17 (0.5 pg/ml), CXCL11 (3.9 pg/ml), CCL20 (4.9 pg/ml) and M-CSF (48 pg/ml). The samples were analysed on a Luminex ${ }^{\circledR 200}{ }^{\mathrm{TM}}$ instrument (Invitrogen, Merelbeke, Belgium), and the data were collected using the xPONENT 3.1 $1^{\mathrm{TM}}$ (Luminex Corporation, Austin, TX, USA) and analysed using the MasterPlex2010 2.0 (MiraiBio Group, Hitachi Solutions America, Ltd., South San Francisco, CA, USA). Intra-assay variance of the method, according to data provided by the manufacturer, is $1.6 \%$ for IFN- $\gamma, 2.1 \%$ for CXCL1, 1.6\% for IL-10, 1.6\% for CCL22, 3.1\% for GM-CSF, 2.2\% for IL-13, 2.2\% for IL-17A, 2.1\% for IL-1RA, 2.0\% for IL-6, 2.6\% for CXCL10, 9.1\% for M-CSF, 2.7\% for CXCL11, 2.1\% for CCL20, 4.8\% for CXCL13, 5.6\% for CCL17 and 1.9\% for CXCL8.

A sandwich enzyme-linked immunosorbent assay (ELISA) was used to measure CCL18. Monoclonal anti-human CCL18 (clone 64507, R\&D Systems, Abingdon, UK) was used for coating and biotinylated anti-human CCL18 antibody (BAF394, R\&D Systems) was used for detection, as previously described (Sandberg et al. 2009). The lower detection limit was $3.9 \mathrm{pg} / \mathrm{ml}$ and samples were analysed in duplicates where samples with CV >15\% were re-analysed.

\subsection{Statistics}

Differences between paired high and low estrogen/progestogen samples were tested within each group by Wilcoxon's signed rank test for cytokines and chemokines (non-normal data distribution) and by paired t-test for gene expression (normal data distribution). Gene expression was analysed as ratios to CD3E for TBX21, GATA3, RORC, FOXP3 and CTLA-4. Testing for statistical differences in base data 
between groups was performed using Chi-2 test. Since the study was designed for comparisons between low and high hormone level phases, we did not perform any statistical comparisons between women with MS and controls. Data were analysed in SPSS 21.0 (IBM Corporation 2012).

\subsection{Ethics}

This study was approved by the Regional Ethical Committee in Linköping (2011/148-31). All women participating in the study gave their oral and written informed consent.

\section{Results}

In total 60 women were included and 13 dropped out leaving 47 women in the statistical analyses (Table 1). Baseline characteristics are presented in Table 1. All MS patients had the relapsingremitting (RR) type of MS. Median EDSS was 1.5 (range 0-3.5) and mean MSSS was 1.45 (range 0.28-6.81). There were no statistical differences in EDSS or MSSS between women using CHC and women not using CHC. All women using CHC were on a 21/7 regimen and no patient reported any missed pill. Most MS patients were on immunomodulatory treatment, similar between the groups except a lower proportion of IFN $\beta$-treated women in the CHC group. Serum concentrations of $17 \beta$ estradiol (E2) and progesterone for the women who were not using hormonal contraception showed expected values in relation to menstrual cycle phase (Table 1).

The $C D 3 E / 18 S$ ratio was significantly lower in the high estrogen/progestogen phase than in the low estrogen/progestogen phase in MS patients using $\mathrm{CHC}(\mathrm{p}=.012)$ and in healthy controls using $\mathrm{CHC}$ $(p=.015)$. Since CD3E, forming a complex with the $\mathrm{T}$ cell receptor, is only expressed in $\mathrm{T}$ cells, it represents a measure of the T cell fraction, which thus seems to fluctuate over the phases in CHC users in line with previous observations (Auerbach et al. 2002). Also, the expression of CD3E remained stable upon $\mathrm{T}$ cell activation (Edström, data to be published) and CD3E was therefore used 
as the reference gene for expression of transcription factors and genes associated with $\mathrm{T}$ cell subsets, thereby normalising data for differences in the size of the $\mathrm{T}$ cell fraction.

Healthy women using CHC showed a small but significant increase in expression of both Th1associated TBX21 (0.67 to 0.81, $\mathrm{p}=0.035)$ and Th2-associated GATA3 (1.0 to $1.1, \mathrm{p}=0.031)$ in the high estrogen/progestogen phase compared with the low estrogen/progestogen phase, and a tendency $(\mathrm{p}=0.054)$ in the same direction was found for TBX21 in women without CHC (Figure 1). Furthermore, a significant decrease in expression of the immune regulatory CTLA-4 was observed in the high estrogen/progestogen phase in healthy controls without CHC $(\mathrm{p}=0.014$, Figure 1$)$. In women with MS, there were no significant differences in gene expression in the high estrogen/progestogen phase compared with the low estrogen/progestogen phase (Figure 2).

In healthy women using $\mathrm{CHC}$ and in both groups of women with MS, the concentrations of the B cellassociated chemokine CXCL13 were significantly higher in the high estrogen/progestogen phase compared with the low estrogen/progestogen phase (Table S1 and Fig 3). In MS patients using CHC, the concentration of IL-13 was lower while in MS patients without hormonal contraception the concentration of IL-10 was higher in the high estrogen/progesterone phase than in the low estrogen/progesterone phase. No other cytokine or chemokine levels were significantly different between high and low estrogen/progestogen phases in any of the four groups (Table S1). Results of CCL20 and M-CSF are omitted since these analytes were not detectable in any of the samples.

\section{Discussion}

Previous findings regarding sex steroids and Th subsets are inconsistent and depend on the context. In studies based on the improvement of autoimmunity during pregnancy, for example in the experimental MS model EAE, estrogens show a protective role and mainly anti-inflammatory actions like decrease in Th1 immunity (Polanczyk et al. 2006). In contrast, studies based on the generally 
stronger immune response in women than in men indicate mainly pro-inflammatory actions of female sex steroids (Maret et al. 2003, Karpuzoglu et al. 2007). Our study is the first to show upregulation of the Th1 and Th2-associated transcription factors during both endogenous and exogenous high estrogen/progestagen exposure. Although the significant changes were modest, they were consistent with the finding of a simultaneously decreased expression of the T-cell inhibitory CTLA-4 gene. To the best of our knowledge, our study is the first to show that sex-steroids in humans may affect the check point regulator CTLA-4. In rodents, it was shown that protective estrogen treatment of EAE was associated with upregulation of CTLA-4 (Matejuk et al. 2002). The discrepancy may be related to difference in species and to levels, duration of sex steroid exposure or route of administration of the steroids. It would be very relevant to further investigate estrogen effects of CTLA-4 in vivo as well as in vitro.

In women with MS, no differences in expression of T cell associated genes were found. When designing the project, we reasoned that the pairwise model, ensuring that patients were on the same dose of the same treatment, would allow us to reveal immune changes in relation to sex steroid cyclicity. However, the most probable explanation for the lack of changes is that the immunomodulatory treatment, which was given to most of the patients, already affected T cell activity and led to a reduced responsiveness to other immunomodulators such as estrogens and progestogens. Although it would be important to further assess cyclic effects of sex steroid hormones in MS, the usually early start of treatment makes it difficult to avoid the effect of immunomodulatory treatment.

Of all the cytokines and chemokines analysed, the only clear finding was a significantly higher plasma concentration of CXCL13 in high estrogen/progestogen phases. CXCL13 acts as a chemoattractant to B cells by binding to its receptor CXCR5, and is involved in homing of B cells and organization of lymphoid tissues (Lalor and Segal 2010). Our finding of high CXCL13 in the high hormonal phase is therefore in line with previous findings of estrogens showing an increased B cell activation and Ig production (Paavonen et al. 1981). Given the presumed beneficial role of estrogen in MS and EAE, the association between CXCL13 and high estrogen/progestogen phases was 
unexpected since CXCL13 has been associated with neuroinflammation in MS (Sellebjerg et al. 2009, Khademi et al. 2013) and EAE (Bagaeva et al. 2006). Assuming that CXCL13 enhances neuroinflammation, the hormone-induced increase in plasma CXCL13 levels could still represent a beneficial effect if increased CXCL13 levels in plasma are associated with decreased levels in the brain or in the cerebrospinal fluid. In line with this scenario it was shown that E2 treatment protected against EAE and led to decreased expression of TNF, IFN- $\gamma$ and IL-17 in the CNS while at the same time these inflammatory cytokines showed increased expression in organs outside the CNS such as peripheral lymphoid organs and spleen (Subramanian et al. 2011). Thus, increased immune activation in the periphery, such as our finding of increased levels of CXCL13 and Th1/Th2-associated gene expression, combined with lower expression of anti-inflammatory CTLA4, may reflect sequestration of immune activity in the periphery linked to a decreased inflammation in the CNS. Although speculative, this interpretation also points to the possibility that sex steroid effects directly in the CNS are responsible for the beneficial effects in MS and EAE. However, the CXCL13 finding needs to be confirmed and further investigated, preferably by simultaneous assessment of peripheral and CNS changes, for example by MRI or by simultaneous measurements in blood and cerebrospinal fluid. Finally, it is also possible that the hormonal concentrations captured at high estrogen/progestogen phases indeed have immune stimulatory effects, which is in line with the stronger immune reactivity noted in women than in men (Klein et al. 2010, Markle and Fish 2014), and the higher incidence in autoimmune diseases like MS in women than in men (D'Hooghe M et al. 2013, Harbo et al. 2013).

There are several confounding factors and there is in general a high inter-individual variation in immune parameters in humans. A strength of our study is therefore the paired sample design, which minimises both these issues. It is important to address the fact that we analysed several cytokines, chemokines, and T cell-associated genes, of which only a few showed significant fluctuations in relation to high and low estrogen/progestogen phases, which could be a result of multiple testing. However, the findings were logical and biologically reasonable (down-regulation of an inhibitor and upregulation of activation markers). In comparison to other studies of immunological fluctuations 
related to sex steroid variations during the menstrual cycle and use of CHC, earlier studies have used both smaller (Faas et al. 2000, Lee et al. 2010) and larger (Arruvito et al. 2007) numbers of participants and our study is the only using a paired design. Furthermore, the choice of cycle days for blood sampling might also influence the outcome. For women using CHC, the samples were drawn on one of the last two days of use of EE/progestogen administration, and on one of the last two hormonefree days occurring after the longest possible period without exposure to EE/progestogen. For the women without hormonal contraception the length of their menstrual cycles may vary, but measurements of estradiol and progesterone confirmed that, as intended, the first sample was drawn in a low estradiol and low progesterone phase and the second in a high estradiol and high progesterone phase. The estrogen/progestogen fluctuations during the menstrual cycle and in $\mathrm{CHC}$ use are much faster than the variations during long term treatment with estriol, which have been shown to have effects on cytokine expression in MS patients (Sicotte et al. 2002), or the changes that occur during pregnancy.

In conclusion, we show that in healthy women, high hormone levels were associated with increases in both Th1- and Th2-associated markers, paralleled by a decreased expression of the inhibitory regulator CTLA-4, a checkpoint of immune activation. These changes were not observed in MS, likely due to ongoing immunomodulatory treatment. In addition, the B cell-associated chemokine CXCL13 was elevated in samples with high versus low hormone levels. Collectively our data indicate mainly immune activating properties of in vivo steroid sex hormone levels associated with CHC and normal menstrual cyclicity.

\section{Aknowledgements}

Petra Cassel, for conducting the Multiplex bead assays and RNA preparations and Karin Söderman, for RNA preparations. Anne-Marie Fornander for performing the CCL18 ELISA and Maria C Jenmalm for valuable suggestions regarding the design of the cytokine/chemokine assays. 
Gunn Johansson, Department of Neurology, University Hospital of Linköping, and Therese Pollack,

Department of Neurology, Karolinska University Hospital of Solna, for recruiting patients.

\section{Funding}

This study was funded by the Swedish Research Council (Grant K2013-61X-22310-01-04) and the County Councils of Östergötland and Västernorrland. The funding sources had no involvement in study design; in the collection, analysis, and interpretation of data; in the writing of the report nor in the decision to submit the article for publication.

\section{References}

Alonso, A., et al., 2005. Recent use of oral contraceptives and the risk of multiple sclerosis. Arch Neurol. 62, 1362-5

Arruvito, L., et al., 2007. Expansion of $c d 4+c d 25+a n d$ foxp3+ regulatory t cells during the follicular phase of the menstrual cycle: Implications for human reproduction. Journal of immunology. $178,2572-8$

Auerbach, L., et al., 2002. Influence of low-dose oral contraception on peripheral blood lymphocyte subsets at particular phases of the hormonal cycle. Fertility and sterility. 78, 83-89

Bagaeva, L.V., et al., 2006. Cxc chemokine ligand 13 plays a role in experimental autoimmune encephalomyelitis. Journal of immunology. 176, 7676-7685

Bernardi, S., et al., 1991. The influence of pregnancy on relapses in multiple sclerosis: A cohort study. Acta neurologica Scandinavica. 84, 403-6

Confavreux, C., et al., 1998. Rate of pregnancy-related relapse in multiple sclerosis. Pregnancy in multiple sclerosis group. The New England journal of medicine. 339, 285-91

D'hooghe M, B., et al., 2013. Female gender and reproductive factors affecting risk, relapses and progression in multiple sclerosis. Gynecol Obstet Invest. 75, 73-84

D'hooghe, M.B., et al., 2012. Menarche, oral contraceptives, pregnancy and progression of disability in relapsing onset and progressive onset multiple sclerosis. J Neurol. 259, 855-861

Edström, M., et al., 2011. Transcriptional characteristics of cd4+ t cells in multiple sclerosis: Relative lack of suppressive populations in blood. Mult Scler J. 17, 57-66

Ernerudh, J., et al., 2011. Regulatory t helper cells in pregnancy and their roles in systemic versus local immune tolerance. American journal of reproductive immunology. 66 Suppl 1, 31-43

Faas, M., et al., 2000. The immune response during the luteal phase of the ovarian cycle: $A$ th2-type response? Fertility and sterility. 74, 1008-1013

Gava, G., et al., 2014. Long-term influence of combined oral contraceptive use on the clinical course of relapsing-remitting multiple sclerosis. Fertility and sterility.

Harbo, H.F., et al., 2013. Sex and gender issues in multiple sclerosis. Therapeutic advances in neurological disorders. 6, 237-48

Hernan, M.A., et al., 2000. Oral contraceptives and the incidence of multiple sclerosis. Neurology. 55, 848-54

Holmqvist, P., et al., 2010. Age at onset of multiple sclerosis is correlated to use of combined oral contraceptives and childbirth before diagnosis. Fertility and sterility. 94, 2835-2837 
Holmqvist, P., et al., 2009. Symptoms of multiple sclerosis in women in relation to cyclical hormone changes. The European journal of contraception \& reproductive health care : the official journal of the European Society of Contraception. 14, 365-70

Karpuzoglu, E., et al., 2007. Ifn-gamma-inducing transcription factor, t-bet is upregulated by estrogen in murine splenocytes: Role of il-27 but not il-12. Mol Immunol. 44, 1808-14

Khademi, M., et al., 2013. Intense inflammation and nerve damage in early multiple sclerosis subsides at older age: A reflection by cerebrospinal fluid biomarkers. PLoS One. 8, e63172

Klein, S.L., Flanagan, K.L., 2016. Sex differences in immune responses. Nat Rev Immunol. 16, 626-38

Klein, S.L., et al., 2010. The xs and y of immune responses to viral vaccines. The Lancet. Infectious diseases. 10, 338-49

Kourtis, A.P., et al., 2014. Pregnancy and infection. The New England journal of medicine. 370, 22118

Kurtzke, J.F., 1983. Rating neurologic impairment in multiple sclerosis: An expanded disability status scale (edss). Neurology. 33, 1444-52

Lalor, S.J., Segal, B.M., 2010. Lymphoid chemokines in the cns. J Neuroimmunol. 224, 56-61

Lee, S., et al., 2010. Fluctuation of peripheral blood t, b, and nk cells during a menstrual cycle of normal healthy women. Journal of immunology. 185, 756-762

Maret, A., et al., 2003. Estradiol enhances primary antigen-specific cd4 t cell responses and th1 development in vivo. Essential role of estrogen receptor alpha expression in hematopoietic cells. European journal of immunology. 33, 512-21

Markle, J.G., Fish, E.N., 2014. Sexx matters in immunity. Trends in immunology. 35, 97-104

Matejuk, A., et al., 2002. Evaluation of the effects of 17beta-estradiol (17beta-e2) on gene expression in experimental autoimmune encephalomyelitis using DNA microarray. Endocrinology. 143, 313-9

Merrill, J.E., et al., 1992. Inflammatory leukocytes and cytokines in the peptide-induced disease of experimental allergic encephalomyelitis in sjl and b10.PI mice. Proceedings of the National Academy of Sciences of the United States of America. 89, 574-8

Morales, L.B., et al., 2006. Treatment with an estrogen receptor alpha ligand is neuroprotective in experimental autoimmune encephalomyelitis. The Journal of neuroscience : the official journal of the Society for Neuroscience. 26, 6823-33

Paavonen, T., et al., 1981. Sex hormone regulation of in vitro immune response. Estradiol enhances human b cell maturation via inhibition of suppressor $t$ cells in pokeweed mitogen-stimulated cultures. J Exp Med. 154, 1935-45

Polanczyk, M., et al., 2003. The protective effect of $17 \beta$-estradiol on experimental autoimmune encephalomyelitis is mediated through estrogen receptor- $\alpha$. Am J Pathol. 163, 1599-1605

Polanczyk, M.J., et al., 2006. Estrogen-mediated immunomodulation involves reduced activation of effector $t$ cells, potentiation of treg cells, and enhanced expression of the pd-1 costimulatory pathway. Journal of neuroscience research. 84, 370-8

Polman, C.H., et al., 2011. Diagnostic criteria for multiple sclerosis: 2010 revisions to the mcdonald criteria. Annals of neurology. 69, 292-302

Pozzilli, C., et al., 1999. Mri in multiple sclerosis during the menstrual cycle: Relationship with sex hormone patterns. Neurology. 53, 622-4

Roxburgh, R.H., et al., 2005. Multiple sclerosis severity score: Using disability and disease duration to rate disease severity. Neurology. 64, 1144-51

Saito, S., et al., 2010. Review article: Th1/th2/th17 and regulatory t-cell paradigm in pregnancy. American journal of reproductive immunology. 63, 601-610

Sandberg, M., et al., 2009. Cord blood cytokines and chemokines and development of allergic disease. Pediatric allergy and immunology : official publication of the European Society of Pediatric Allergy and Immunology. 20, 519-27

Sellebjerg, F., et al., 2009. Increased cerebrospinal fluid concentrations of the chemokine cxcl13 in active ms. Neurology. 73, 2003-10 
Sena, A., et al., 2012. Oral contraceptive use and clinical outcomes in patients with multiple sclerosis. J Neurol Sci. 317, 47-51

Sicotte, N.L., et al., 2002. Treatment of multiple sclerosis with the pregnancy hormone estriol. Ann Neurol. 52, 421-428

Subramanian, S., et al., 2011. Oestrogen-mediated protection of experimental autoimmune encephalomyelitis in the absence of foxp3+ regulatory t cells implicates compensatory pathways including regulatory b cells. Immunology. 132, 340-347

Thorogood, M., Hannaford, P.C., 1998. The influence of oral contraceptives on the risk of multiple sclerosis. British journal of obstetrics and gynaecology. 105, 1296-9

Voskuhl, R.R., et al., 2016. Estriol combined with glatiramer acetate for women with relapsingremitting multiple sclerosis: A randomised, placebo-controlled, phase 2 trial. Lancet neurology. 15, 35-46

Zorgdrager, A., De Keyser, J., 1997. Menstrually related worsening of symptoms in multiple sclerosis. J Neurol Sci. 149, 95-97

Zorgdrager, A., De Keyser, J., 2002. The premenstrual period and exacerbations in multiple sclerosis. Eur Neurol. 48, 204-6 
Table 1. Age and parity of the four groups of women in the study, estradiol and progesterone concentrations of the women without combined hormonal contraception (CHC), and immunomodulatory medication of the women with MS. (ns = not significant)

\begin{tabular}{|c|c|c|c|c|c|c|}
\hline & & 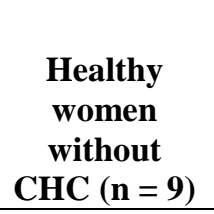 & $\begin{array}{c}\text { Healthy } \\
\text { women } \\
\text { with CHC } \\
(\mathrm{n}=13)\end{array}$ & $\begin{array}{c}\text { MS } \\
\text { patients } \\
\text { without } \\
\text { CHC } \\
(\mathbf{n}=13)\end{array}$ & $\begin{array}{c}\text { MS } \\
\text { patients } \\
\text { with } \\
\text { CHC } \\
(\mathbf{n}=12)\end{array}$ & $\mathbf{p}$ \\
\hline Age, Median (range) & & $27(21-42)$ & $24(21-40)$ & $36(25-39)$ & $27(22-40)$ & .002 \\
\hline Parity, Median (range) & & $0(0-4)$ & $0(0-2)$ & $0(0-2)$ & $0(0-4)$ & ns \\
\hline \multirow{2}{*}{$\begin{array}{l}\text { Estradiol (pmol/L), } \\
\text { Mean (SD) }\end{array}$} & Cycle Day 5-7 & 124 (39) & & $130(121)$ & & ns \\
\hline & Cycle Day 26-28 & $522(365)$ & & $360(251)$ & & ns \\
\hline \multirow{2}{*}{$\begin{array}{l}\text { Progesterone (nmol/L) } \\
\text { Mean (SD) }\end{array}$} & Cycle Day 5-7 & $1.9(0.7)$ & & $1.5(0.8)$ & & ns \\
\hline & Cycle Day 26-28 & $25(22)$ & & 17 (13) & & ns \\
\hline \multirow{6}{*}{$\begin{array}{l}\text { Immunomodulatory } \\
\text { Medication (n) }\end{array}$} & Fingolimod & & & 0 & 1 & ns \\
\hline & Glatiramer & & & 0 & 1 & ns \\
\hline & IFN- $\beta$ & & & 8 & 3 & .030 \\
\hline & Natalizumab & & & 4 & 3 & ns \\
\hline & Rituximab & & & 0 & 1 & ns \\
\hline & No medication & & & 1 & 3 & ns \\
\hline
\end{tabular}


A

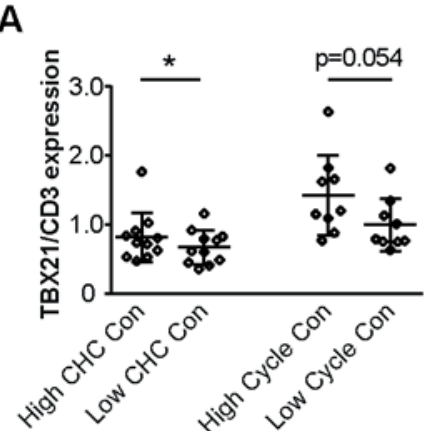

B

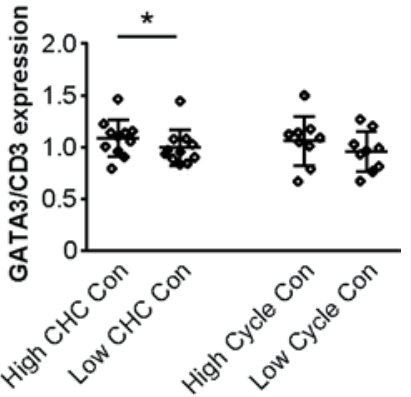

C

D
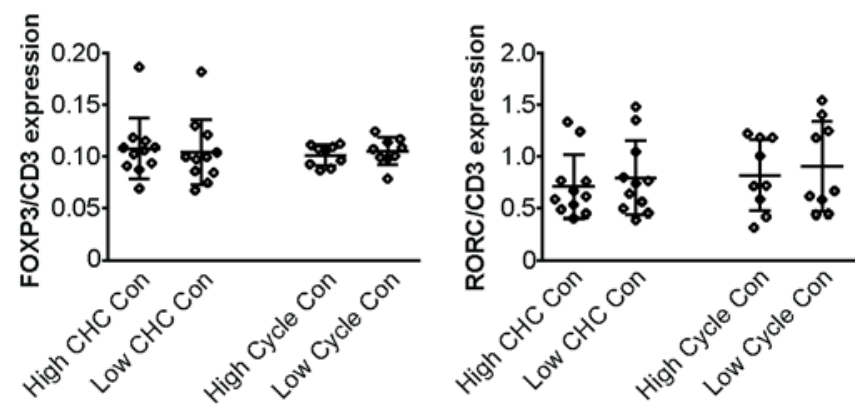

$\mathrm{E}$

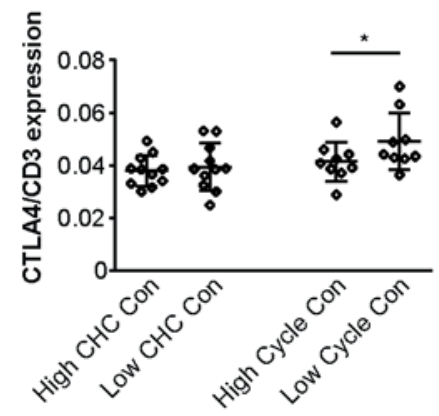

Figure 1. Expression of subset-specific transcription factors and CTLA4 in healthy women with or without CHC.

Within-group comparisons of TBX21 (A), GATA3 (B), RORC (C), FOXP3 (D) and CTLA4 (E) in paired high versus low estrogen/progestogen phases in healthy women (Con) with combined hormonal contraception (CHC) and with natural menstrual cycle (Cycle). Gene expression is presented as a ratio to $C D 3 E$ expression (mean and SD). Statistical testing using paired t-test. ${ }^{*} \mathrm{p}<$ .05 

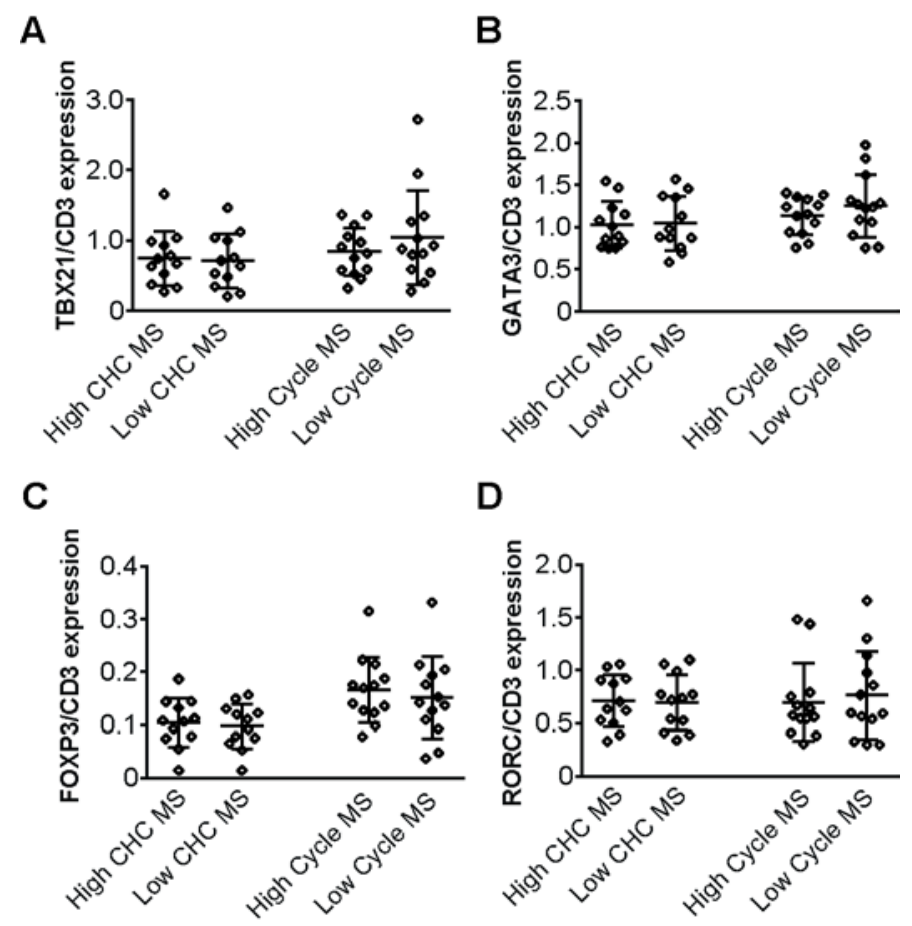

D
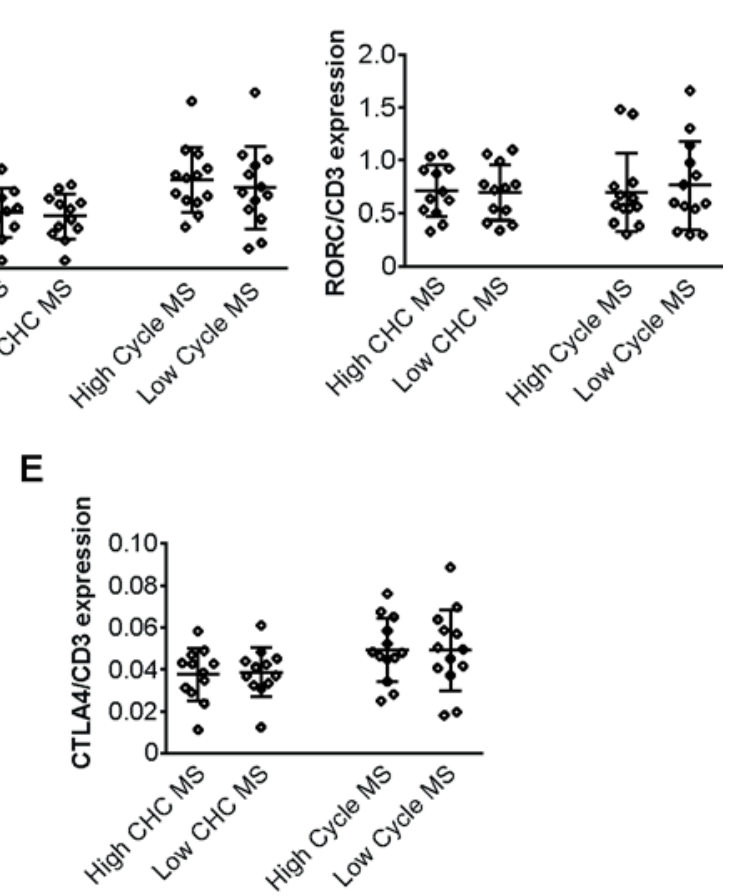

Figure 2. Expression of subset-specific transcription factors and CTLA4 in female MS patients with or without CHC.

Within-group comparisons of TBX21 (A), GATA3 (B), RORC (C), FOXP3 (D) and CTLA4 (E) in paired high versus low estrogen/progestogen phases in MS patients (MS) with combined hormonal contraception (CHC) and with natural menstrual cycle (Cycle). Gene expression is presented as a ratio to $C D 3 E$ expression (mean and SD). Statistical testing using paired t-test. * $\mathrm{p}<.05$ 


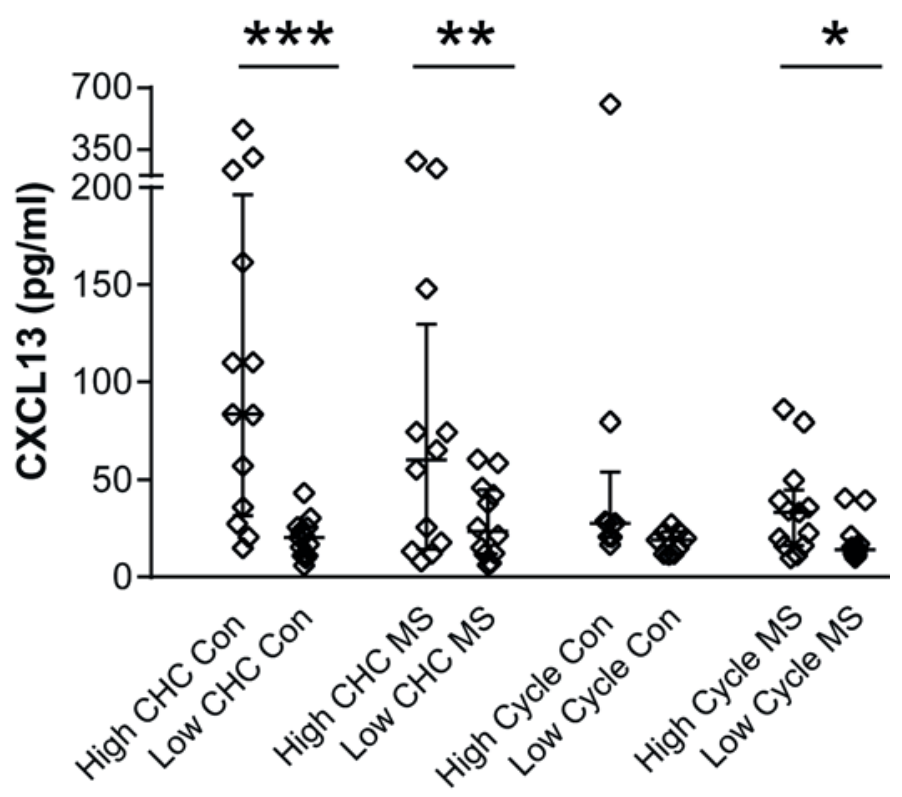

Figure 3. Levels of CXCL13 in study subjects.

The concentration of CXCL13 in plasma was measured and compared in high versus low estrogen/progestogen phases in MS patients (MS) and healthy women (Con) with combined hormonal contraception (CHC) and with natural menstrual cycle (Cycle). Concentrations are presented as median and $25^{\text {th }}$ and $75^{\text {th }}$ percentiles. Hypothesis testing using Wilcoxon's signed rank test. ${ }^{*} \mathrm{p}<.05$, $* * \mathrm{p}<.01, * * * \mathrm{p}<.001$ 
Table S1. Median plasma concentrations $\mathrm{pg} / \mathrm{ml}$ (interquartile range) of cytokines and chemokines in high

\begin{tabular}{|c|c|c|c|c|c|c|}
\hline & \multicolumn{3}{|c|}{ MS patients using $\mathrm{CHC}$} & \multicolumn{3}{|c|}{ MS patients without hormonal contraception } \\
\hline & $\mathrm{Hi}$ & Lo & $\mathrm{p}$ & $\mathrm{Hi}$ & Lo & $\mathrm{p}$ \\
\hline IL6 & $5.2(0.8-26)$ & $9.3(1.6-24)$ & 0.21 & $7.8(2.2-21)$ & $7.6(4.7-21)$ & 0.51 \\
\hline IL-1RA & $58(43-86)$ & $59(43-146)$ & 0.43 & $54(33-94)$ & $59(42-81)$ & 0.75 \\
\hline GM-CSF & $20(13-22)$ & $26(16-48)$ & 0.11 & $12(9.0-24)$ & $15(10-21)$ & 0.65 \\
\hline CXCL10 & $407(270-621)$ & $411(295-677)$ & 0.43 & $468(312-1204)$ & $533(353-1014)$ & 0.92 \\
\hline CXCL11 & $30(13-63)$ & $27(17-77)$ & 0.25 & $21(12-86)$ & $21(14-54)$ & 0.81 \\
\hline IFN-Y & $6.9(4.2-15)$ & $12(4.1-19)$ & 0.97 & $8.8(6.2-28)$ & $9.9(4.1-30)$ & 0.86 \\
\hline CCL22 & 902 (828-1030) & 933 (857-1091) & 0.1 & 875 (749-1026) & $822(731-1010)$ & 0.86 \\
\hline CCL17 & $11(6.5-19)$ & $11(8.5-23)$ & 0.16 & $9.0(6.2-14)$ & $8.7(6.2-12)$ & 0.22 \\
\hline IL-13 & $1.6(1.6-6.1)$ & $5.7(1.6-14)$ & $.03^{*}$ & $7.3(1.6-25)$ & $7.9(1.6-19)$ & 0.07 \\
\hline CXCL1 & 376 (284-451) & $352(270-408)$ & 0.24 & $293(186-495)$ & $293(113-417)$ & 0.2 \\
\hline IL-17A & $4.7(3.2-14)$ & $7.9(2.3-12)$ & 0.93 & $4.6(3.2-11)$ & $5.0(3.0-12)$ & 0.97 \\
\hline CXCL8 & $3.7(2.6-12)$ & $5.1(2.4-12)$ & 0.88 & $4.7(2.8-7.8)$ & $5.5(2-0-11)$ & 0.35 \\
\hline IL-10 & $4.2(1.6-5.9)$ & $7.8(1.6-12)$ & 0.21 & $5.4(1.6-15)$ & $4.7(1.6-8.0)$ & $.047^{*}$ \\
\hline CXCL13 & $60(14-130)$ & $23(12-45)$ & $.01^{*}$ & $33(16-45)$ & 14 (11-19) & $.02^{*}$ \\
\hline CCL18 & 29 (17-39) & $27(16-52)$ & 0.53 & $23(19-32)$ & $25(18-36)$ & 0.6 \\
\hline
\end{tabular}


(Hi) and low (Lo) estrogen/progestogen phases in the four groups of women studied. Statistical dif1

\begin{tabular}{ccccc}
\multicolumn{2}{c}{ Healthy women using CHC } & & Healthy women without hormonal cor \\
\hline $\mathrm{Hi}$ & $\mathrm{LO}$ & $\mathrm{p}$ & $\mathrm{Hi}$ & Lo \\
$9.8(0.8-15)$ & $7.4(2.7-14)$ & 0.51 & $11(5-7-19)$ & $6.6(3.1-31)$ \\
$64(41-95)$ & $81(29-120)$ & 0.75 & $84(57-154)$ & $78(34-173)$ \\
$18(11-31)$ & $24(10-36)$ & 0.46 & $16(11-37)$ & $13(6.3-28)$ \\
$279(254-294)$ & $269(250-359)$ & 0.25 & $243(207-492)$ & $263(191-424)$ \\
$21(13-28)$ & $16(13-39)$ & 0.65 & $16(11-32)$ & $21(16-31)$ \\
$15(5.2-20)$ & $15(5.7-26)$ & 0.53 & $31(6.3-65)$ & $39(5.7-90)$ \\
$997(628-1074)$ & $899(754-1160)$ & 0.25 & $1194(906-1438)$ & $1017(892-1620)$ \\
$10(3.7-17)$ & $12(5.7-19)$ & 0.51 & $16(11-23)$ & $12(8.3-25)$ \\
$12(16-29)$ & $13(2.6-22)$ & 0.89 & $12(3.8-14)$ & $12(2.7-13)$ \\
$349(227-48)$ & $347(184-441)$ & 0.92 & $404(210-499)$ & $364(327-523)$ \\
$7.5(3.8-10)$ & $8.5(3.4-11)$ & 0.12 & $13(3.1-18)$ & $11(2-4-26)$ \\
$5.3(2.7-7.8)$ & $5.7(3.3-8.3)$ & 0.86 & $5.3(3.7-11)$ & $5.9(3.0-12)$ \\
$9.1(4.3-12)$ & $8.6(3.4-11)$ & 0.6 & $5.6(3.5-12)$ & $4.9(2.5-12)$ \\
$83(32-196)$ & $20(11-26)$ & $.001 *$ & $28(21-54)$ & $19(12-23)$ \\
$31(22-38)$ & $39(21-49)$ & 0.31 & $39(21-49)$ & $32(23-57)$
\end{tabular}


ferences were tested by Wilcoxon's signed rank test.

traception

0.89

0.68

0.09

0.95

0.21

0.16

0.59

0.52

0.4

0.31

0.21

0.77

0.48

0.07

0.37 